\title{
OP-0134 - SNPs in PRKCA-HIF1A-GLUT1 are associated with diabetic kidney disease in a Chinese Han population with type 2 diabetes
}

\author{
Yan Huang, Li Jin, Cheng Hu
}

\begin{abstract}
Shanghai Diabetes Institute, Shanghai Key Laboratory of Diabetes Mellitus, Shanghai Clinical Center for Diabetes, Shanghai Jiao Tong University Affiliated Sixth People's Hospital, 600 Yishan Road, Shanghai, 200233, China
\end{abstract}

BACKGROUND: Diabetic kidney disease has been confirmed to have genetic underpinnings. Diabetes complications depend on abnormal intracellular glucose accumulation transferred by glucose transporters. Studies have shown that the glucose transporter 1 (GLUTI) allele (XbaI-) is associated with nephropathy in type 2 diabetes. The association of hypoxia-inducible factor 1A (HIF1A) and diabetic kidney disease has been reported. Protein kinase $\mathrm{C}$ alpha (PRKCA) inhibitors have been a focus of research on diabetic kidney disease treatment. Moreover, there is evidence showing that PRKCA, HIF1A and GLUTI are located in a pathway closely related to hypoxic-related kidney injury. Therefore, we carried out this experiment to explore the relationship between SNPs in PRKCA-HIF1A-GLUT1 and diabetic kidney disease in Chinese Han people.

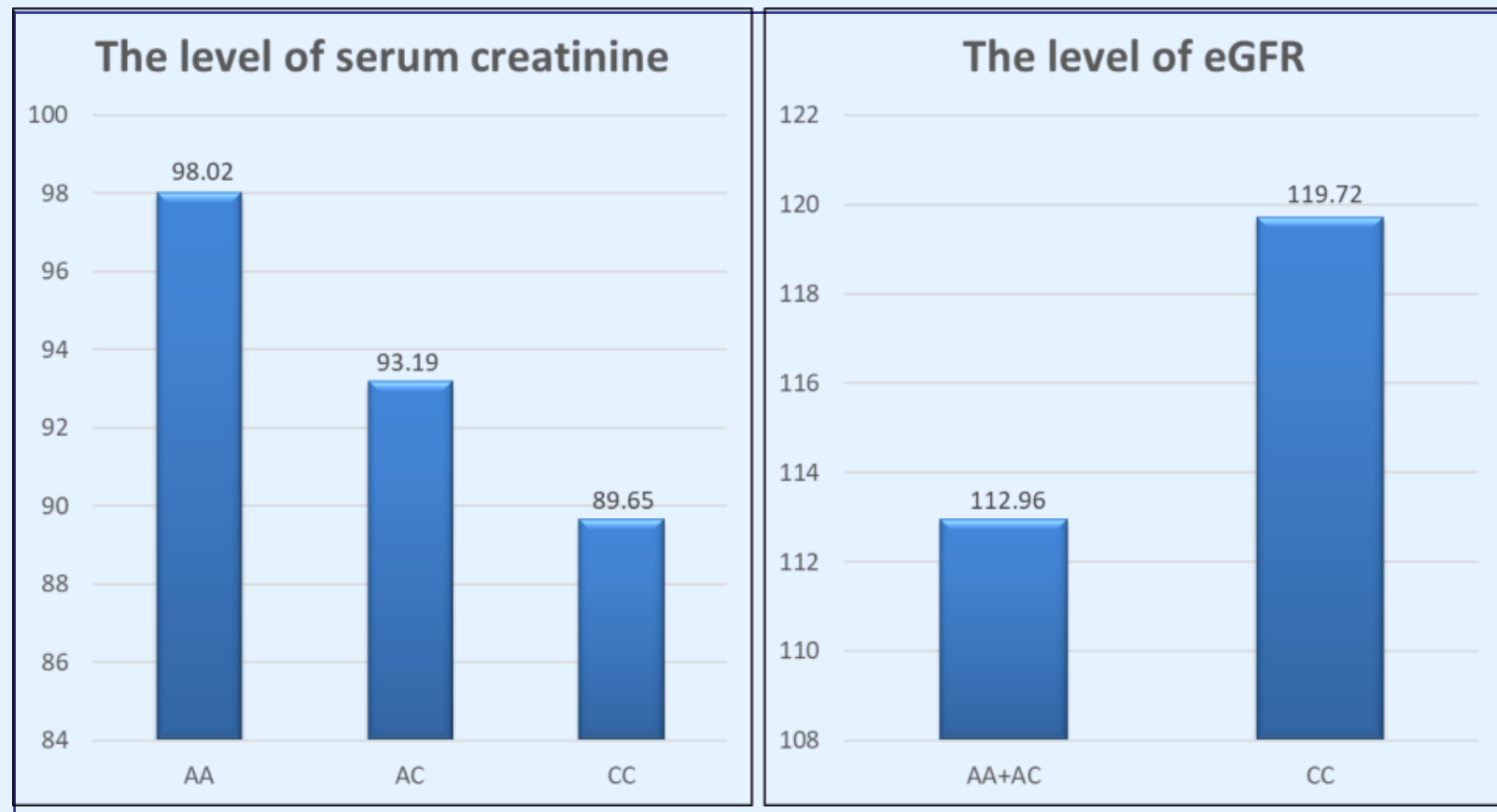

Different genotypes at rs1681851 have different mean serum creatinine and eGFR

\begin{tabular}{|c|c|c|c|c|c|c|c|c|c|c|c|c|c|}
\hline$C H R$ & SNP & Position & Gene & Allele & $M A F$ & Model & $O R$ & $S E$ & $\boldsymbol{P}$ & Model & $O R$ & $S E$ & $\boldsymbol{P}$ \\
\hline \multirow[t]{2}{*}{1} & rs1681851 & 42923357 & GLUT1 & $\mathrm{A} / \mathrm{C}$ & 0.4934 & 1 & 1.149 & 0.0690 & 0.0443 & 2 & 1.104 & 0.0747 & 0.1846 \\
\hline & & & & & & 3 & 1.331 & 0.1118 & 0.0105 & 4 & 1.084 & 0.1141 & 0.4780 \\
\hline \multirow[t]{2}{*}{14} & rs2301108 & 61730746 & HIF $1 A$ & $\mathrm{~A} / \mathrm{G}$ & 0.1754 & 1 & 1.169 & 0.0884 & 0.0771 & 2 & 1.289 & 0.0964 & 0.0085 \\
\hline & & & & & & 3 & 1.222 & 0.1058 & 0.0580 & 4 & 1.139 & 0.2495 & 0.6011 \\
\hline \multirow[t]{2}{*}{14} & rs116908431 & 61738919 & HIFlA & $\mathrm{G} / \mathrm{T}$ & 0.0529 & 1 & 1.048 & 0.1537 & 0.7610 & 2 & 1.101 & 0.1681 & 0.5679 \\
\hline & & & & & & 3 & 1.079 & 0.1584 & 0.6317 & 4 & 0.289 & 1.1860 & 0.2956 \\
\hline \multirow[t]{2}{*}{14} & rs79865957 & 61744249 & HIF $1 A$ & $\mathrm{~T} / \mathrm{G}$ & 0.1594 & 1 & 1.128 & 0.0923 & 0.1919 & 2 & 1.263 & 0.1007 & 0.0204 \\
\hline & & & & & & 3 & 1.179 & 0.1078 & 0.1262 & 4 & 0.998 & 0.2788 & 0.9929 \\
\hline \multirow[t]{2}{*}{17} & rs 9915504 & 66794316 & PRKCA & $\mathrm{A} / \mathrm{G}$ & 0.3359 & 1 & 0.894 & 0.0723 & 0.1222 & 2 & 0.919 & 0.0787 & 0.2813 \\
\hline & & & & & & 3 & 0.821 & 0.0984 & 0.0446 & 4 & 0.978 & 0.1512 & 0.8833 \\
\hline
\end{tabular}

METHODS: A total of 2552 participants from Shanghai Diabetes Institute Inpatient Database of Shanghai Jiao Tong University Affiliated Sixth People's Hospital were involved in the stage 1 crosssectional population. A total of 6015 subjects from the Hong Kong Diabetes Register were included for validation. Genotyping of participants was conducted by the MassARRAY Compact Analyzer (Agena Bioscience, San Diego, CA, USA). The data was analyzed by plink, SAS, Haploview.

RESULTS: We identified variants associated with diabetic kidney disease in stage 1. Rs1681851 ( $\mathrm{P}=0.0105, \mathrm{OR}=1.331)$ in GLUT1 as well as rs2301108 $(\mathrm{P}=0.0085, \mathrm{OR}=1.289)$ and $\mathrm{rs} 79865957(\mathrm{P}=0.0204$, $\mathrm{OR}=1.263$ ) in HIFlA were significantly associated with diabetic kidney disease. Regarding DKD-related traits, rs1681851 was associated with plasma creatinine levels $(\mathrm{P}=0.0169$, beta $=4.822)$ and eGFR ( $\mathrm{P}=0.0457$, beta $=-6.956)$. Moreover, the results showed the interactions between PRCKA-GLUT1 in the occurrence of DKD. We further sought validation of the 8 suggestive SNPs in a prospective cohort and found that rs900836 was associated with the percentage change in eGFR slope. We performed a meta-analysis of case-control analysis data from the Hong Kong samples together with the stage 1 data from Shanghai. No SNP reached statistical significance in the meta-analysis.

CONCLUSION: Our results suggest potential association between SNPs in PRKCA-HIF1A-GLUT1 and diabetic kidney disease in Chinese Han people. 